\title{
Errata: Spectral hole burning for ultrasound-modulated optical tomography of thick tissue
}

\author{
Xiao Xu* \\ Sri-Rajasekhar Kothapalli*, $\dagger$ \\ Honglin Liu \\ Lihong V. Wang \\ Washington University in St. Louis \\ Department of Biomedical Engineering \\ Optical Imaging Laboratory \\ St. Louis, Missouri 63130 \\ [DOI: 10.1117/1.3533983]
}

This article [J. Biomed. Opt. 15, 066018 (2010)] was originally published online on 13 December 2010 with an error in the author affiliations. The author Sri-Rajasekhar Kothapalli completed the work for this paper while affiliated with Washington University in St. Louis, but is now currently affiliated with Stanford University, as shown here.

All versions of the article were corrected on 16 December 2010. The article appears correctly in print.

\footnotetext{
*Authors contributed equally.

$\dagger$ Current address: Stanford University, Department of Radiology, Molecular Imaging Program at Stanford and Bio-X Program, Palo Alto, California 94305.

Address all correspondence to: Lihong V. Wang, Optical Imaging Laboratory, Department of Biomedical Engineering, Washington University in St. Louis, One Brookings Dr., Campus Box 1097, St. Louis, MO 63130. Tel: 314-935-6152; Fax: 314-935-7448; E-mail: Ihwang@biomed.wustl.edu.
} 